\title{
VLBI monitoring of two distant quasars as a showcase for 'EVN Lite'
}

\author{
S. Frey ${ }^{* \dagger},{ }^{a}$ O. Titov, ${ }^{b}$ A. Melnikov, ${ }^{c}$ P. de Vicente ${ }^{d}$ and F. Shu ${ }^{e}$ \\ ${ }^{a}$ Konkoly Observatory, MTA Research Centre for Astronomy and Earth Sciences, Budapest, \\ Hungary \\ ${ }^{b}$ Geoscience Australia, Canberra, Australia \\ ${ }^{c}$ Institute of Applied Astronomy, Russian Academy of Sciences, Saint Petersburg, Russia \\ ${ }^{d}$ Observatorio de Yebes (IGN), Yebes, Spain \\ e Shanghai Astronomical Observatory, Chinese Academy of Sciences, Shanghai, China \\ E-mail: frey.sandorecsfk.mta.hu, oleg.titovega.gov.au, aemeiaaras.ru, \\ p.devicentedoan.es, sfceshao.ac.cn
}

\begin{abstract}
Two prominent quasars at extremely high redshifts, J0906+6930 $(z=5.47)$ and $\mathrm{J} 2102+6015(z=$ 4.57) have been monitored with an ad-hoc array of five very long baseline interferometry (VLBI) stations (Badary, Svetloe, Zelenchukskaya, Yebes, and Sheshan) at 2.3 and $8.6 \mathrm{GHz}$ in the past two years. The primary purpose of this series of 24-hour astrometric-style experiments was to investigate possible changes of the accurate source positions over time. Moreover, the apparent non-variability of the objects allowed us to combine data obtained at multiple epochs and produce the best-quality VLBI images of the quasars at these observing frequencies available so far. Here we present the imaging results. We argue that this type of observations could be attractive for the proposed 'EVN Lite' concept in the future where a subset of the telescopes in the European VLBI Network (EVN) would observe more frequently than the regular EVN sessions.
\end{abstract}

14th European VLBI Network Symposium \& Users Meeting (EVN 2018)

8-11 October 2018

Granada, Spain

* Speaker.

${ }^{\dagger} \mathrm{SF}$ thanks for the support received from the Hungarian Research, Development and Innovation Office (OTKA NN110333), and from the China-Hungary Collaboration and Exchange Programme by the International Cooperation Bureau of the Chinese Academy of Sciences. 


\section{Introduction}

Radio-loud active galactic nuclei (AGNs) in the early Universe are rare. The distant quasars J0906+6930 at redshift $z=5.47$ [6] and J2102+6015 at $z=4.57$ [7] stand out from the known sample [2] with their compact emission on milliarcsecond (mas) angular scale with high (0.1-Jy level) flux densities measured at $\mathrm{GHz}$ radio frequencies. This makes them ideal targets for very long baseline interferometry (VLBI) observations.

VLBI imaging can reveal pc-scale radio structure of AGN jets by providing the finest angular resolution currently achievable in astronomy. On the other hand, VLBI is also successfully employed for astrometric and geodetic studies. A unique capability of the technique is to accurately monitor Earth rotation and orientation with respect to a quasi-inertial reference frame [1] realized by the positions of distant, compact radio AGNs. Ideal astrometric reference sources have compact, nearly unresolved mas-scale structure in VLBI images and as little apparent proper motion as possible.

\section{Observations and imaging results}

A series of regular 1-day VLBI sessions is being performed to determine the accurate astrometric positions of the two high-redshift quasars (J0906+6930 and J2102+6015) and their possible changes over a longer period of time (O. Titov et al., in prep.). The quasars are observed along with a large set of bright compact VLBI reference sources. Here we show the results of an imaging analysis based on observations conducted at 5 different epochs spanning nearly 7 months, from February to August 2017. More details of the observations, data analysis and results are presented in [3]. The participating radio telescopes were Badary, Svetloe, Zelenchukskaya (Russia), Yebes (Spain), and Sheshan (China), providing interferometer baselines longer than $9000 \mathrm{~km}$, i.e. on a global scale. The scheduling, observations, and correlation were performed following an agreement between the Institute of Applied Astronomy of the Russian Academy of Sciences (IAA RAS) and Geoscience Australia, with the collaboration of the Yebes Observatory in Spain and the Shanghai Astronomical Observatory in China. The data were correlated at the IAA RAS in Saint Petersburg with the DiFX software correlator. Note that while the participating radio telescopes are all members of the European VLBI Network (EVN), these observations were arranged independently from the EVN, outside its regular sessions.

We obtained sensitive high-resolution VLBI images of J0906+6930 and J2102+6015 at two observing frequencies, 2.3 and $8.6 \mathrm{GHz}$. The apparent flux density invariability of the quasars allowed us to combine the data taken at all 5 epochs, leading to an excellent $(u, v)$ coverage and high-quality images.

J0906+6930 is one of the highest redshift blazars known. Our 2.3-GHz map is the first-ever VLBI image obtained at a frequency below $8 \mathrm{GHz}$. The observations reveal a sharply bent helical inner jet structure (Fig. 1) within $\sim 3$ mas from the core, first suggested by [9]. According to the brightness distribution modelling, the core has a high brightness temperature $\left(\sim 2 \times 10^{11} \mathrm{~K}\right)$ indicating Doppler-boosted emission. The jet is closely inclined to our line of sight, as expected from a blazar. J0906+6930 seems to have a helical mas-scale jet that we see in projection in the sky. 

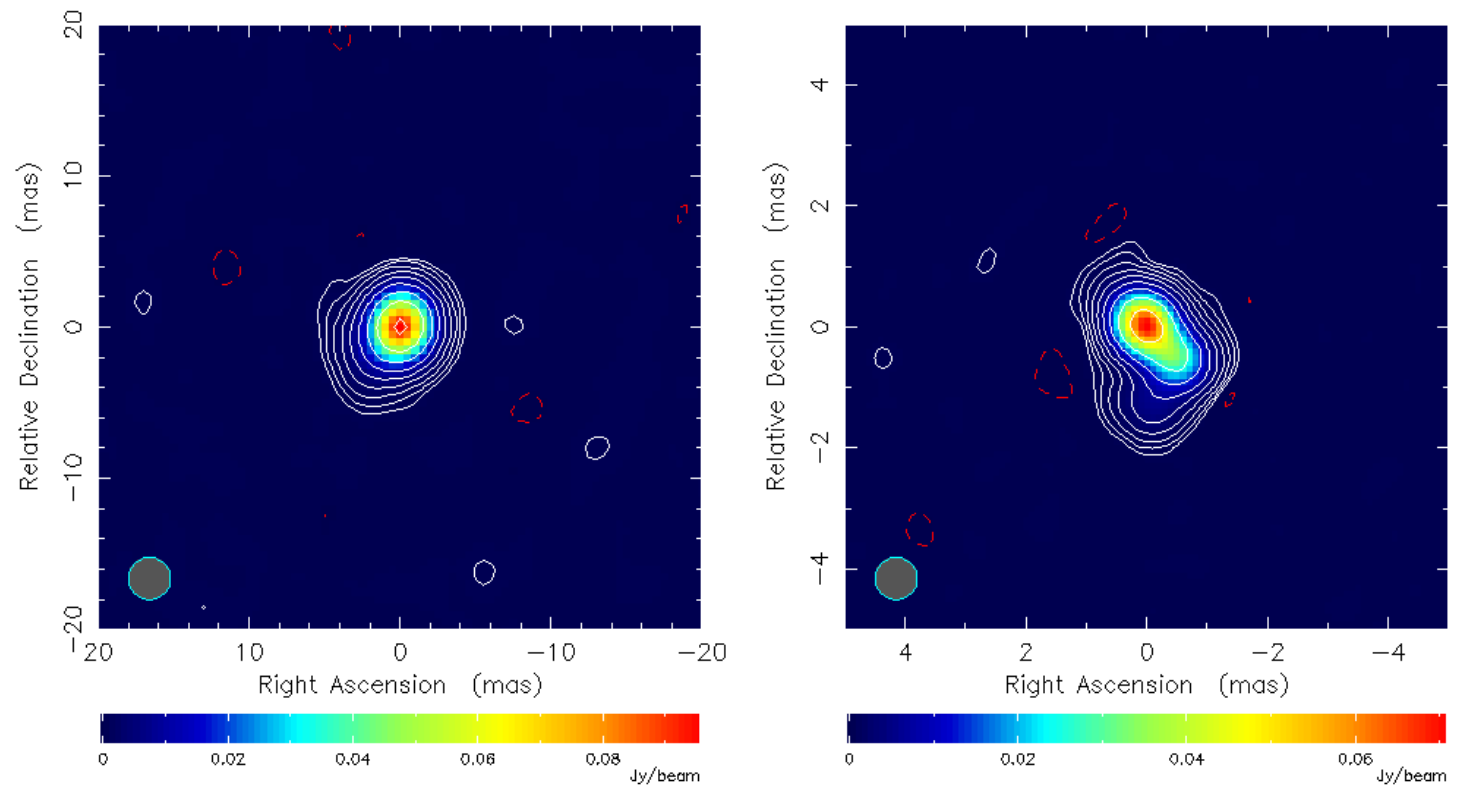

Figure 1: 2.3-GHz (left) and 8.6-GHz (right) VLBI images of J0906+6930 [3]. The peak brightnesses are $95.6 \mathrm{mJy}$ beam $^{-1}$ and $70.9 \mathrm{mJy}$ beam $^{-1}$ at 2.3 and $8.6 \mathrm{GHz}$, respectively. The first contours are drawn at $\sim 3 \sigma$ image noise levels ( $\pm 0.35 \mathrm{mJy}$ beam $^{-1}$ at $2.3 \mathrm{GHz}$, and $\pm 0.2 \mathrm{mJy}_{\text {beam }}^{-1}$ at $8.6 \mathrm{GHz}$ ). The positive contour levels increase by a factor of 2 . The circular Gaussian restoring beams are shown in the lower-left corners. The full widths at half maximum (FWHM) are 2.8 mas and 0.7 mas at 2.3 and $8.6 \mathrm{GHz}$, respectively.
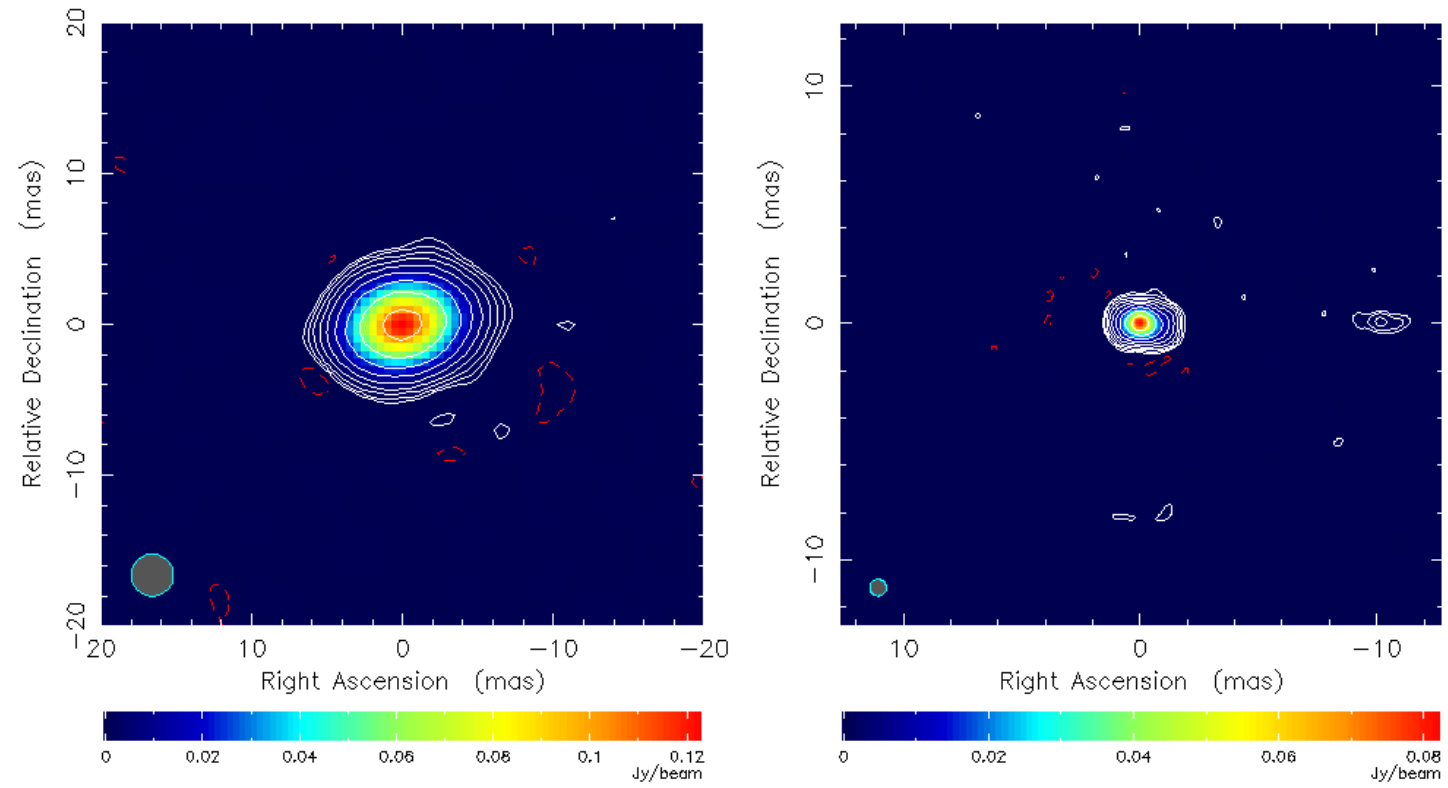

Figure 2: 2.3-GHz (left) and 8.6-GHz (right) VLBI images of J2102+6015 [3]. The peak brightnesses are $123.3 \mathrm{mJy}$ beam $^{-1}$ and $82.4 \mathrm{mJy}_{\text {beam }}{ }^{-1}$ at 2.3 and $8.6 \mathrm{GHz}$, respectively. The first contours are drawn at $\sim 3 \sigma$ image noise levels $\left( \pm 0.4 \mathrm{mJy}_{\text {beam }}{ }^{-1}\right.$ at $2.3 \mathrm{GHz}$, and $\pm 0.22 \mathrm{mJy}_{\text {beam }}^{-1}$ at $8.6 \mathrm{GHz}$ ). The positive contour levels increase by a factor of 2 . The circular Gaussian restoring beams are shown in the lower-left corners. The FWHM are 2.8 mas and 0.7 mas at 2.3 and $8.6 \mathrm{GHz}$, respectively. 
J2102+6015 shows an elongated radio structure in the east-west direction within the innermost 2 mas. It can be described with a symmetric three-component brightness distribution model at 8.6 $\mathrm{GHz}$ (Fig. 2). Based on the imaging and model-fitting, there is no evidence for Doppler-boosting in this powerful gigahertz-peaked spectrum source [3].

\section{An example for 'EVN Lite' projects}

This type of observations involving a limited number of radio telescopes for a day-long snapshot imaging of high-declination sources could be attractive for the 'EVN Lite' (or 'EVN Light') concept [4] in the future. This observing mode has been proposed for a couple of years but is not yet available for the users. The basic idea is to use a subset of EVN telescopes that would be available for more frequent observations in VLBI mode than the regular EVN sessions that usually take place 3 times a year. In our case, we made use the non-variability of the target sources to prepare sensitive images by combining the individual data sets taken several months apart. Similar weeklymonthly time sampling could in turn be useful to monitor rapid structural changes and flux density variations in other bright compact radio sources. Thus for this type of projects, 'EVN Lite' sessions organized at least once in a month would be beneficial. For bright astrometric reference sources like the ones observed here, sensitivity is not an issue, considering the current EVN capabilities.

Apart from monitoring large samples of sources, which is often difficult to perform with oversubscribed arrays like the full EVN and the Very Long Baseline Array (VLBA), other potential use cases of the sub-network partly overlap with those of e-EVN [8]. In particular, the availability of an array for observing radio transients [5] would be an attractive application, if sufficiently flexible proposing, scheduling and timely correlation are offered. A benefit of 'EVN Lite' compared to other VLBI arrays (VLBA, the East-Asia VLBI Network or the Australian Long Baseline Array) would be the truly global baselines and thus the achievable high angular resolution, as was the case in our ad-hoc experiments reported here. Clearly, a quick-response VLBI facility like this would not be able to compete with large arrays in e.g. collecting area, sensitivity, dense $(u, v)$ coverage, and the choice of observing fequencies and modes. But a flexible, responsive EVN sub-array that is relatively easy to access for the users would open up the possibility for new types of science experiments. Ultimately, the needs and the proposal pressure from a wide user community should drive the organizational and technical details of a prospective 'EVN Lite'.

\section{References}

[1] P. Charlot, ICRF3, the new realization of the International Celestial Reference Frame, in proceedings of 14th European VLBI Network Symposium \& Users Meeting, PoS (EVN2 018 ) 136 (2019)

[2] R. Coppejans, S. Frey, D. Cseh, et al., On the nature of bright compact radio sources at $z>4.5$, MNRAS 463 (2016) 3260 [arXiv: 1609.00575 ].

[3] S. Frey, O. Titov, A. Melnikov, P. de Vicente, F. Shu, High-resolution radio imaging of two luminous quasars beyond redshift 4.5, A\&A 618 (2018) A68 [arXiv: 1807.06837 ].

[4] M. Lindqvist, A. Szomoru, Present status and technical directions of the EVN, in proceedings of 12th European VLBI Network Symposium and Users Meeting, POS (EVN 2014 ) 031 (2015) 
[5] Z. Paragi, Transient Science with the e-EVN, in proceedings of 13th European VLBI Network Symposium and Users Meeting, Transactions of IAA RAS 40 (2017) 99 [arXiv: 1612.00508 ].

[6] R. W. Romani, D. Sowards-Emmerd, L. Greenhill, P. Michelson, Q0906+6930: The Highest Redshift Blazar, ApJ 610 (2004) L9 [arXiv: astro-ph/ 0406252$].$

[7] D. Sowards-Emmerd, R. W. Romani, P. F. Michelson, J. S. Ulvestad, Blazar Counterparts for 3EG Sources at $-40^{\circ}<$ Decl. $<0^{\circ}$ : Pushing South through the Bulge, ApJ 609 (2004) 564 [arXiv:astro-ph/0406252].

[8] A. Szomoru, EXPReS and the e-EVN, in proceedings of 9th European VLBI Network Symposium and Users Meeting, POS (IX EVN Symposium) 040 (2009)

[9] Y. Zhang, T. An, S. Frey, et al., J0906+6930: a radio-loud quasar in the early Universe, MNRAS 468 (2017) 69 [arXiv:1702.03925]. 